\title{
The Transcription Factor Rvl453 Regulates the Expression of qor and Confers Resistant to Clofazimine in Mycobacterium tuberculosis
}

\author{
Yuanyuan Li \\ Lei $\mathrm{Fu}$ \\ Weiyan Zhang \\ Xi Chen \\ Yu Lu
}

Beijing Key Laboratory of Drug Resistance Tuberculosis Research, Department of Pharmacology, Beijing Tuberculosis and Thoracic Tumor Research Institute, Beijing Chest Hospital, Capital Medical University, Beijing, I0I I49, People's Republic of China
Correspondence: Yu Lu

Beijing Key Laboratory of Drug

Resistance Tuberculosis Research,

Department of Pharmacology, Beijing

Tuberculosis and Thoracic Tumor

Research Institute, Beijing Chest Hospital,

Capital Medical University, Beiguan Street,

Tongzhou District, Beijing, People's

Republic of China

Tel +86 10-8950-9358

Email luyu4876@hotmail.com
Objective: Clofazimine plays an important role in the treatment of drug-resistant tuberculosis. However, the mechanism of clofazimine resistance remains unclear. In order to slow down the occurrence of clofazimine resistance, it is necessary to study its resistance mechanism.

Methods: In this study, we constructed Rv1453 knockout, complementary and overexpressed strain. The minimum inhibitory concentration (MIC) of clofazimine against Mycobacterium tuberculosis was detected by microplate alamar blue assay (MABA). The transcription levels of $R v 1453$ and its adjacent genes were detected by quantitative reverse transcriptase PCR. The purified Rv1453 protein was used for electrophoretic mobility shift assay (EMSA) to identify the binding site of Rv1453 protein.

Results: The minimum inhibitory concentration (MIC) of clofazimine increased about 4-fold for the $R v 1453$ knockout strain and decreased about 4-fold for the Rv1453 overexpressed strain compared with Mycobacterium tuberculosis H37Rv. Further analysis showed that Rv1453 protein, as a regulatory protein, binds to the RNA polymerase binding site of qor and blocks the transcription process.

Conclusion: This study preliminarily revealed that Rv1453 protein of Mycobacterium tuberculosis affects its susceptibility to clofazimine by regulating the transcription level of qor, which is shedding a new light on the mechanism of clofazimine resistance.

Keywords: resistance mechanisms, transcriptional regulation, electrophoretic mobility shift assay, redox

\section{Introduction}

Tuberculosis (TB) is a chronic infectious disease caused by the bacillus Mycobacterium tuberculosis and the leading cause of death from a single infectious disease (ranking above HIV/AIDS). Estimated 10 million people worldwide were infected with TB in 2019 , and the number of new infections per year has declined slowly in recent years, but not fast enough to reach the target of a 20\% reduction between 2015 and 2020 . The global treatment success rate of multidrug resistant (MDR)/rifampicin resistant (RR) tuberculosis is only $57 \% .{ }^{1}$ Due to the long course of treatment and high cost of drugresistant tuberculosis, it brings a heavy burden to families and society. Clofazimine (CFZ) was initially discovered in the development of new antituberculosis drugs. With further studies, it was found that CFZ can also be used for the treatment of drugresistant TB. ${ }^{2,3}$ The World Health Organization designated it as group B drug for the treatment of drug-resistant tuberculosis in $2018 .^{4}$ 
According to current researches, the mechanism of CFZ resistance is mainly related to the efflux pump. And the reported genes associated with CFZ resistance were Rv0678, Rv1979c and Rv2535c. ${ }^{5-7}$ The specific mechanism of CFZ has not been fully elucidated. By using a fraction of the membrane isolated from M. smegmatis, it was confirmed that CFZ appeared to compete with menaquinone for electrons. This is the initiating event in the respiratory chain of $M$. tuberculosis, through which reduced CFZ undergoes spontaneous oxidation to generate reactive oxygen species, such as superoxide and hydrogen peroxide. ${ }^{8}$ This hypothesis was supported by another study, which claimed that the addition of high concentrations of menaquinone to the culture medium antagonized the antibacterial activity of CFZ. ${ }^{9}$ Furthermore, inactivating the cytochrome bd-type quinol oxidase in the mycobacterial respiratory chain increased the susceptibility of M. smegmatis to CFZ, which seems to be consistent with inhibition of bacterial respiration. ${ }^{10}$ The authors speculated that the protective effect of cytochrome bd is mediated by neutralization or inhibition of reactive oxygen species generated by CFZ.

In our previous study, we detected genes associated with clofazimine resistance by whole-genome sequencing from 18 strains of M. tuberculosis. We found that the mutation frequencies of $R v 0678, R v 1979 c, R v 2535 c$ were $36.4 \%(4 / 11), 18.2 \%(2 / 11)$ and $0 \%(0 / 11)$, respectively. However, the mutation frequency of the Rv1453 intergenic region was $54.5 \%(6 / 11)$. In this study, we constructed $R v 1453$ knockout strain, $R v 1453$ complementary strain and $R v 1453$ overexpressed strain to explore the correlation between $R v 1453$ and clofazimine resistance. The $R v 1453$ gene encodes the $46.6 \mathrm{KDa}$ transcriptional regulatory protein. And qor gene encodes quinone oxidoreductase, which is adjacent to $R v 1453$. We speculated that Rv1453mediated resistance to clofazimine was related to the reduction of reactive oxygen species. The accumulation of quinones in cells could produce a range of toxic effects. Quinones are highly redox active molecules and can generate reactive oxygen radicals during the spontaneous reduction to semiquinone and hydroquinone. Reactive oxygen species (ROS) can cause oxidative damage to DNA, proteins and lipids, and stimulate oxidative stress. ${ }^{11}$ Quinone oxidoreductases (QOR) have protective and detoxifying effects on cells by reducing quinones to hydroquinone, which could bind to glucuronic acid or sulfate and be excreted. It has been reported that quinone spontaneously loses one electron and becomes semiquinone, whereas in the presence of QOR, quinone can be reduced to hydroquinone by catalyzing the transfer reaction of two electrons, thus avoiding the generation of free radicals. ${ }^{12-14}$ In this study, we investigated that Rv1453 transcriptional regulatory protein could change the transcriptional level of qor gene to affect the sensitivity of the strain to clofazimine.

\section{Materials and Methods}

\section{Bacteria Strains and Culture Conditions}

M. tuberculosis H37Rv (ATCC 27294) and recombinant strains were grown at $37^{\circ} \mathrm{C}$ in $7 \mathrm{H} 9$ liquid medium supplemented with $0.2 \%$ glycerin, $0.05 \%$ Tween 80 , and $10 \%$ oleic acid-albumin-dextrose-catalase (OADC) or on $7 \mathrm{H} 10$ solid medium with $0.5 \%$ glycerin and $10 \%$ OADC. E. coli strains were grown at $37^{\circ} \mathrm{C}$ in liquid $\mathrm{LB}$, liquid $2 \mathrm{YT}$ or on solid LB medium.

\section{Construction of Recombinant Strains}

The $R v 1453$ knockout strain was constructed as previously reported. ${ }^{15}$ The Rv1453 gene of M. tuberculosis H37Rv was replaced with res-sacB-hyg-res gene cassette by allelic exchange method. Briefly, chromosomal sequences flanking Rv1453 were amplified by PCR from the genomic DNA of M. tuberculosis H37Rv. Primers LFP and LRP, which contained the Van91I site, respectively, were used to amplify the left arm of Rv1453. Primers RFP and RRP, which contained the Van91I site, respectively, were used to amplify the right arm of Rv1453. These fragments were cloned into the vector p0004s. The resultant plasmid was ligated with the plasmid phAE159 by the PacI site. The phasmid was electroporated into M. smegmatis $\mathrm{mc}^{2} 155$ to generate the recombinant phages. M. tuberculosis $\mathrm{H} 37 \mathrm{Rv}$ cells were infected with the recombinant phages at $37^{\circ} \mathrm{C}$ and hygromycin-resistant (HygR) colonies were screened. The $R v 1453$ overexpressed strain was constructed by electroporation with pMV361 carrying a $1266 \mathrm{bp}$ segment of the Rv1453 open reading frame inserted between HindIII and ClaI restriction sites. The Rv1453 complementary strain was constructed by transforming the recombinant pMV361 to the Rv1453 knockout strain. The recombinant strains were verified by RT-PCR. The primers used in this study are listed in Tables $\mathrm{S} 1$ and $\underline{\mathrm{S} 2}$.

\section{MIC Determination}

The MIC of clofazimine against $M$. tuberculosis was determined by MABA. ${ }^{16}$ The concentration of clofazimine ranges 
from 10 to $0.005 \mathrm{mg} / \mathrm{L}$ by using two-fold dilutions method. M. tuberculosis $\mathrm{H} 37 \mathrm{Rv}$ cultured to an exponential phase was added to the 96-well plates containing different concentrations of clofazimine. The color of each well was recorded the following day after the addition of Alamar blue and Tween 80. The MIC was defined as the lowest drug concentration that prevented the color change (from blue to pink).

\section{Total RNA Extraction}

Total RNA of $M$. tuberculosis $\mathrm{H} 37 \mathrm{Rv}$ and recombinant strains were extracted from freshly cultured bacteria by using the QIAgen RNA mini kit (QIAgen, Germany) according to the manufacturer's protocol. We used the kit in combination with RNAprotect Bacteria Reagent to ensure reliable gene expression analysis and Lysing Matrix B to achieve complete disruption and homogenization.

\section{Quantitative Reverse Transcriptase PCR}

The RNA was reverse transcribed to cDNA using the PrimeScript RT reagent kit with gDNA Eraser (TaKaRa, Japan). The primers listed in Table S1 were used, and the RT-PCR was performed in a $20 \mu \mathrm{L}$ PCR solution from the TB Green Premix Ex Taq II (TaKaRa, Japan) according to the manufacturer's protocol. StepOnePlus real-time PCR system (Applied Biosystems, America) was used to perform the RT-PCR with PCR conditions of $95^{\circ} \mathrm{C}$ for 1 minute, then 40 cycles at $95^{\circ} \mathrm{C}$ for 5 seconds, $63.4^{\circ} \mathrm{C}$ for 30 seconds, and $72^{\circ} \mathrm{C}$ for 30 seconds. The RNA polymerase sigma factor $\operatorname{sig} A$ was used as an internal control to normalize the level of target genes for each individual sample. The relative gene expression was calculated using the $2^{-\Delta \Delta \mathrm{Ct}}$ method and we used the logarithm of the relative gene expression for the algorithm analysis. The clofazimine drug concentration we used in this experiment was $0.06 \mu \mathrm{g} / \mathrm{mL}$, and the time points were set as $6 \mathrm{~h}$, $16 \mathrm{~h}$, and $52 \mathrm{~h}$ for the dosed group and the blank control group, respectively.

\section{Rv|453 Protein Expression and Purification in E. coli BL2I (DE3)}

The Rv1453 gene was amplified by PCR from M. tuberculosis $\mathrm{H} 37 \mathrm{Rv}$ genome using the forward and reverse primers, which contained the NdeI and HindIII restriction sites, respectively. We sequenced the DNA fragment cloned into the $\mathrm{T}$ vector. We then cloned it to the expression vector pET30a to generate the His tag fusion protein. The recombinant plasmid (pET30a
+Rv1453) was transformed into E. coli BL21 (DE3) and grown in $2 \mathrm{YT}$ liquid medium at $37^{\circ} \mathrm{C}$ in an orbital shaker at $200 \mathrm{rpm}$ to an optical density at $600 \mathrm{~nm}$ (OD600) of 0.6-0.8. The expression of Rv1453 was induced by the addition of isopropyl-D-1-thiogalactopyranoside at a final concentration of $0.5 \mathrm{mM}$ and grown for an additional $4 \mathrm{~h}$. Cells were harvested by centrifugation at $5000 \mathrm{rpm} / \mathrm{min}$ for 5 minutes. His-tagged Rv1453 was purified with Capturem $^{\text {TM }}$ His-Tagged Purification Kit (TaKaRa, Japan) according to the manufacturer's protocol. The protein was purified with $8 \mathrm{M}$ urea under denaturing conditions. Purity of the His6-Rv1453 protein was identified by Western blot, and protein concentration was determined by using the BCA Protein Assay Kit (Solarbio, China).

\section{Electrophoretic Mobility Shift Assay (EMSA)}

The upstream regions (-500) of $R v 1455$ and qor gene were amplified by PCR using gene-specific primers. The PCR products were purified by using the MiniBEST DNA segment Purification Kit.

The binding reaction mixture $(10 \times)$ contained 100 $\mathrm{mmol} / \mathrm{L}$ Tris- $\mathrm{HCl}, 500 \mathrm{mmol} / \mathrm{L} \mathrm{KCl}, 10 \mathrm{mmol} / \mathrm{L}$ DTT, $50 \%$ glycerol, $10 \mathrm{mmol} / \mathrm{L}$ EDTA. The Rv1453 protein and DNA segment were incubated at $37^{\circ} \mathrm{C}$ for 1.5 hours. Then, the samples were separated by the native PAGE gel in an ice bath containing $1 \times$ Tris-borate-EDTA at $110 \mathrm{~V}$ for 1 hour. The native PAGE gel was stained with SYBR Green I dye for about 15 minutes and placed in an ultraviolet analyzer to observe the bands.

We also used the 3 'biotin-labeled DNA probes to identify the binding sites. Both the forward and reverse chains of the Rv1455 probe were labeled with biotin, but only the forward chain of the qor probes was labeled with biotin. Electrophoretic mobility shift assays (EMSAs) were carried out according to manufacturer's protocol for the LightShift ${ }^{\mathrm{TM}}$ Chemiluminescent EMSA Kit (Thermo Fisher, America). The binding reaction mixture contained $10 \mathrm{mmol} / \mathrm{L}$ Tris- $\mathrm{HCl}, 50 \mathrm{mmol} / \mathrm{L} \mathrm{KCl}, 1 \mathrm{mmol} / \mathrm{L}$ DTT, $5 \%$ glycerol, $1 \mathrm{mmol} / \mathrm{L}$ EDTA and $50 \mu \mathrm{g} / \mathrm{mL}$ poly (dI-dC). For the Rv1455 probe, the binding reactions of single-chain probe to Rv1453 protein and double-chain probe to Rv1453 protein were detected, respectively.

\section{Computational Analysis}

The virtual footprint (http://www.prodoric.de/vfp/) and softBerry (http://www.softberry.com/berry.phtml?topic= 
bprom\&group $=$ programs\&subgroup $=$ gfindb) software were used to identify the Rv1453 protein-binding motif.

\section{Statistical Analysis}

The relative expression levels of $R v 1453$ gene and its adjacent genes in recombinant strains were compared with M. tuberculosis $\mathrm{H} 37 \mathrm{Rv}$ and were tested using a single sample $t$ test. Two-way analysis of variance was used to compare the relative expression levels of various genes among different strains under the action of clofazimine, and the M. tuberculosis H37Rv under the action of clofazimine was taken as a reference. Statistical analysis software was GraphPad prism 8.0 and $\mathrm{P}<0.05$ was considered statistically different.

\section{Results}

\section{Construction and Validation of Recombinant Strains}

The recombinant plasmid pMV361+Rv1453 and pET30a + Rv1453 has been validated by enzyme digestion and DNA sequencing (Figure S1). Then, we compared the relative expression levels of $R v 1453$ gene in the $R v 1453$ knockout strain, $R v 1453$ complementary strain, $R v 1453$ overexpressed strain and M. tuberculosis $\mathrm{H} 37 \mathrm{Rv}$ to verify the recombinant strains (Figure 1). Compared with the M. tuberculosis $\mathrm{H} 37 \mathrm{Rv}$, the relative expression level of $R v 1453$ gene in the knockout strain was significantly decreased, while that in the complementary strain and the overexpressed strain were significantly increased. Therefore, it was confirmed that the Rv1453 knockout strain, the $R v 1453$ complementary strain, and the $R v 1453$ overexpressed strain were successfully constructed.

\section{Susceptibility of Recombinant Strains to Clofazimine}

MABA method was used to detect the MIC of isoniazid, rifampicin and clofazimine against $M$. tuberculosis $\mathrm{H} 37 \mathrm{Rv}$, $R v 1453$ knockout strain, $R v 1453$ complementary strain and $R v 1453$ overexpressed strain (Table 1). We found that, compared with the M. tuberculosis H37Rv, the MIC value of clofazimine to the knockout strain was increased by about 4-fold, while decreased by about 4-fold for the overexpressed strain. As for the complementary strain, it was restored to the same level as that of the M. tuberculosis H37Rv. If hygromycin $(75 \mu \mathrm{g} / \mathrm{mL})$ was added to the knockout strain, the MIC of clofazimine against the knockout strain could be restored to the normal level. At the same

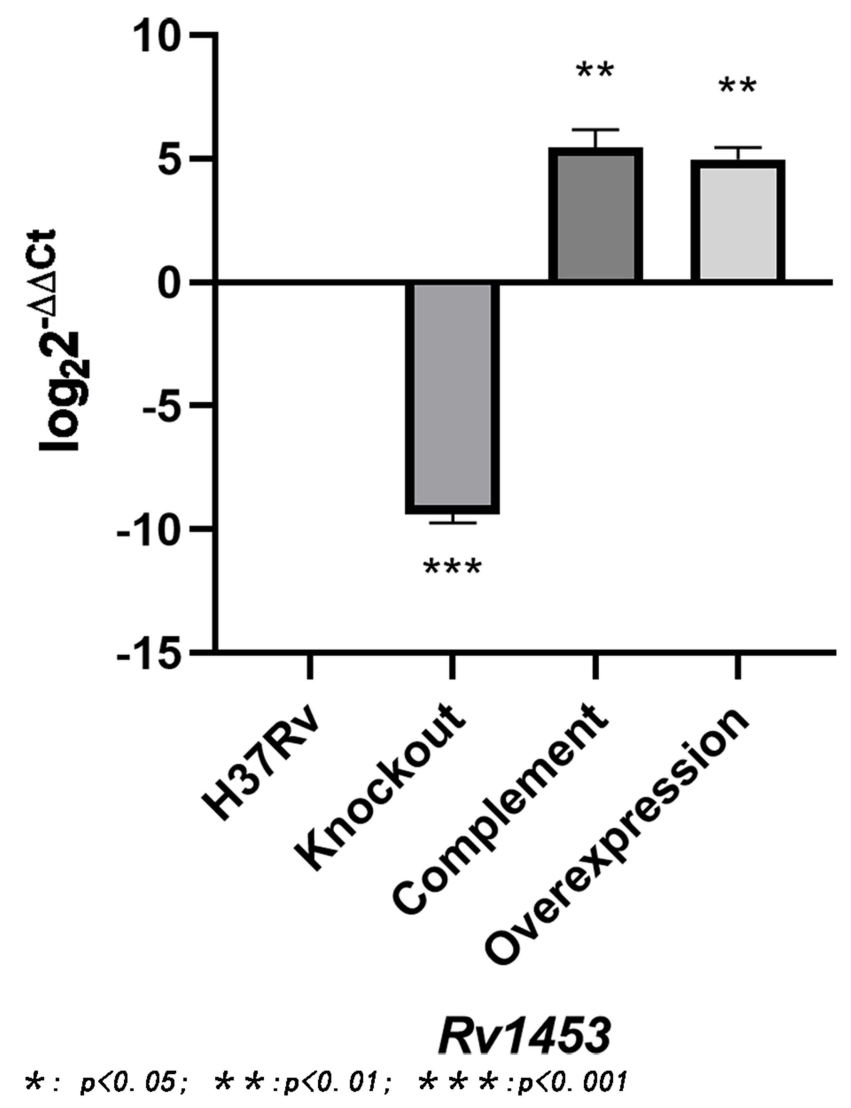

Figure I The relative expression level of $R v / 453$ gene in the recombinant strains.

time, it was found that the MIC of isoniazid and rifampicin could also be increased against the $R v 1453$ knockout strain, while the MIC of that against complementary strain and overexpressed strain were normal. The MIC of isoniazid and rifampicin against the knockout strain could be reduced to the normal level when added with hygromycin.

\section{The Relative Expression Level of Rvl453} Adjacent Genes in the Recombinant

\section{Strains}

We have found that $R v 1453$ affects the sensitivity of the strain to clofazimine. $R v 1453$ encodes the transcriptional

Table I MIC of Anti-Tuberculosis Drugs Against the Recombinant Strains and H37Rv $(\mu \mathrm{g} / \mathrm{ml})$

\begin{tabular}{|l|l|l|l|}
\hline & INH & RFP & CFZ \\
\hline H37Rv & 0.040 & 0.010 & 0.156 \\
Knockout & 5.000 & 0.040 & 0.625 \\
Knockout (with hygromycin) & 0.040 & 0.020 & 0.156 \\
Complement & 0.040 & 0.020 & 0.078 \\
Overexpression & 0.040 & 0.010 & 0.040 \\
\hline
\end{tabular}


regulatory protein, and the relative expression levels of its adjacent genes were detected by RT-PCR to further investigate its transcriptional regulatory function (Figure 2). It was found that the relative expression levels of $R v 1455$ and qor gene were significantly increased in both the knockout and complementary strains but slightly decreased in the overexpressed strain.

\section{Effects of Clofazimine on the Relative Expression Levels of Rvl453 Gene and Its Adjacent Genes in Recombinant Strains}

RT-PCR results showed that the relative expression of $R v 1455$ gene and qor gene was significantly upregulated when $R v 1453$ gene was knockout, while slightly down regulated if $R v 1453$ gene was overexpressed. In order to further study the effect of clofazimine on the transcription regulation function of Rv1453 protein, the relative expression levels of $R v 1453$ gene and its adjacent genes in the recombinant strains were detected after clofazimine treatment at 6, 16 and 52 hours, respectively. With the increase in drug treatment time, the relative expression level of $R v 1455$ gene and qor gene in $R v 1453$ knockout strain continued to increase slightly, while that decreased in Rv1453 complementary strain and $R v 1453$ overexpressed strain at $52 \mathrm{~h}$ after drug treatment (Figure 3).

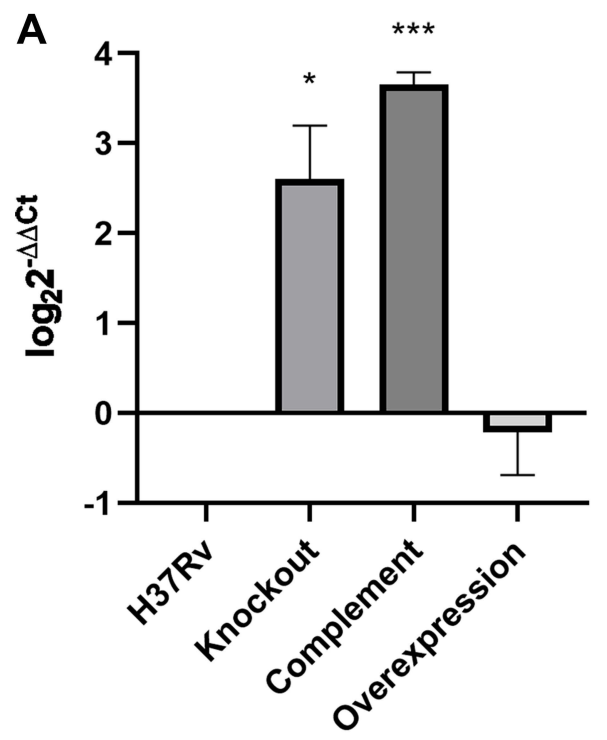

Rv1455

$\star: p<0.05 ; * *: p<0.01 ; * \star \star: p<0.001$

\section{Purification and Validation of Rvl453} Protein

In order to further explore how Rv1453 protein plays a negative regulatory role in the transcription of $R v 1455$ gene and qor gene, we expressed Rv1453 protein in E. coli and purified it, then detected its purity by Western blot (Figure 4). Under denaturation condition, we finally obtained the purified Rv1453 protein and the molecular weight of the protein is approximately $48 \mathrm{kDa}$.

\section{Transcriptional Regulation Mechanism of RvI 453 Protein on Its Neighboring Genes} According to the predicted sites that may bind to Rv1453 protein, the primers were designed, and the DNA segments of the qor gene promoter region and the Rv1455 gene promoter region were amplified by PCR, respectively. The renatured Rv1453 protein was then used to test whether it could bind to the qor promoter region or the Rv1455 promoter region (Figure 5). It showed that Rv1453 protein could bind to them, and if the amount of protein is too small, the binding reaction is not obvious.

To explore the DNA binding sites that could bind to Rv1453 protein, two qor double-stranded probes ( -325 to $-301 ;-254$ to -230 ) were designed, with biotin-labeled on the forward strand, respectively. We also designed three Rv1455 probes ( -176 to -139$)$, one was double-stranded

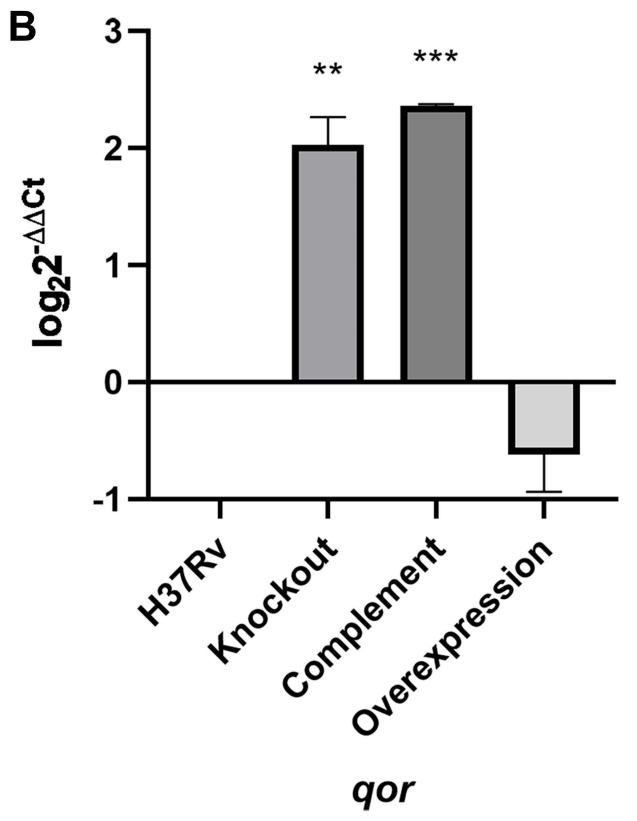

Figure 2 The relative expression levels of the adjacent genes of $R v / 453$ in the recombinant strain. (A) The relative expression levels of $R v / 455$ in the recombinant strain. (B) The relative expression levels of qor in the recombinant strain. 
A

Rv1453

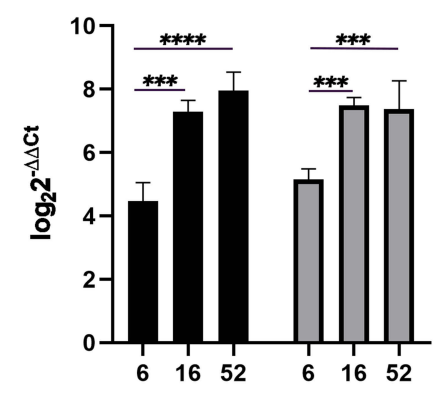

Time (Hours)
B

Rv1455

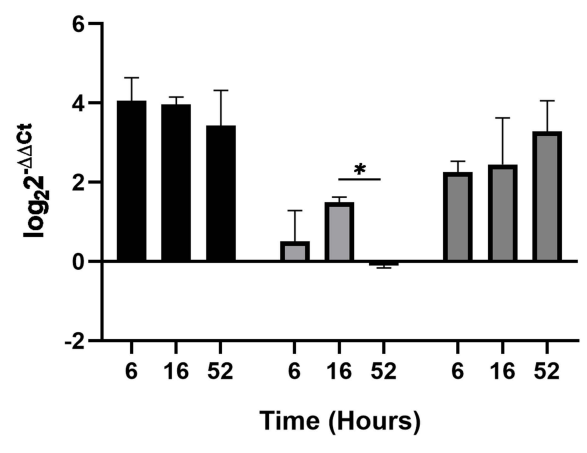

Complementary

口 Overexpressed

口 Knockout $* * * *: p<0.0001$

- Complementary

Overexpressed

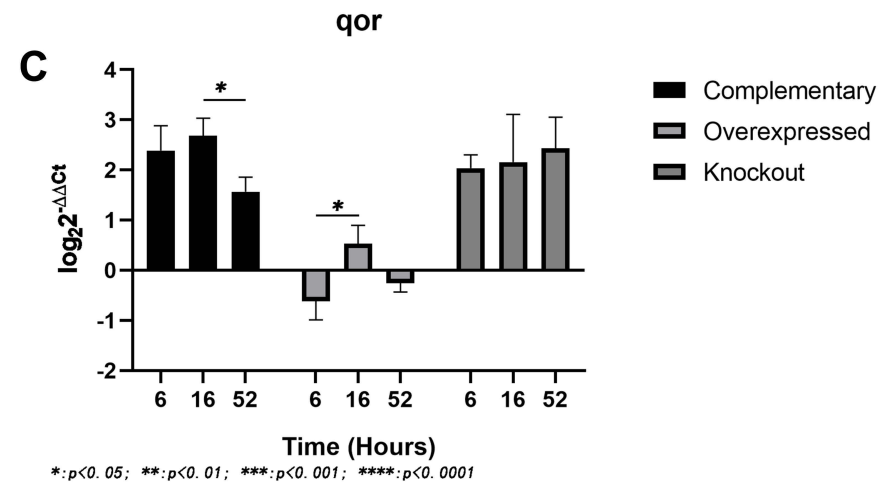

Figure 3 Relative expression levels of Rv 1453 gene (A) and its adjacent genes (B and $\mathbf{C}$ ) in the recombinant strains under the action of clofazimine. M: protein marker, Lane I: pET30a flowthrough, Lane 2: pET30a + RvI453 flowthrough, Lane 3: pET30a wash, Lane 4: pET30a + Rv1453 wash, Lane 5: pET30a eluate, Lane 6: pET30a + Rv1453 eluate (nondenaturing), Lane 7: pET30a + Rvl453 eluate I (denatured), Lane 8: pET30a + Rv I453 eluate 2 (denatured), and Lane 9: pET30a + RvI453 eluate 3 (denatured).

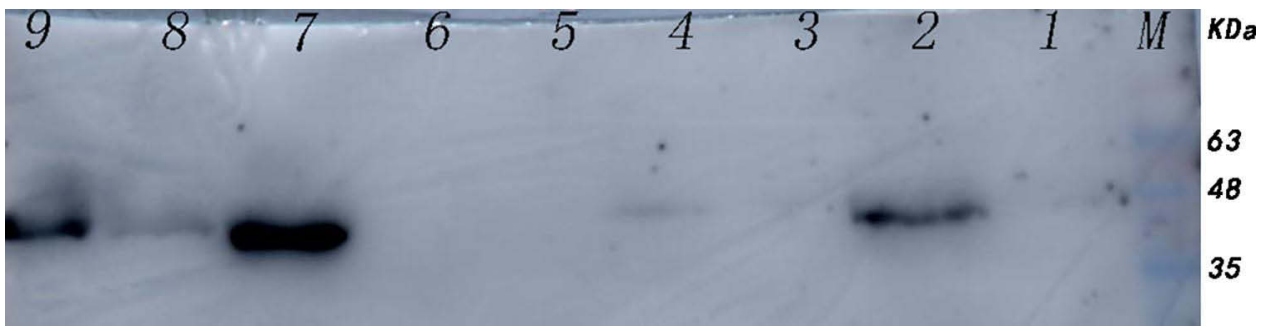

Figure 4 Western blotting of Rv1453 protein. M: DL500bp DNA Marker, lanel: segments qor gene promoter region, lane2: segment of qor promoter region and Rv1453 protein mixture (DNA: protein=1:5), lane3: segment of Rv/455 gene promoter region, lane4: segment of Rv/455 promoter region and Rvl453 protein mixture (DNA: protein=1:5), lane5: segment of qor promoter region and Rvl453 protein mixture (DNA: protein=10:3), lane6: segment of Rv/455 promoter region and Rv1453 protein mixture (DNA: protein=10:3). The arrows indicate the shift bands.

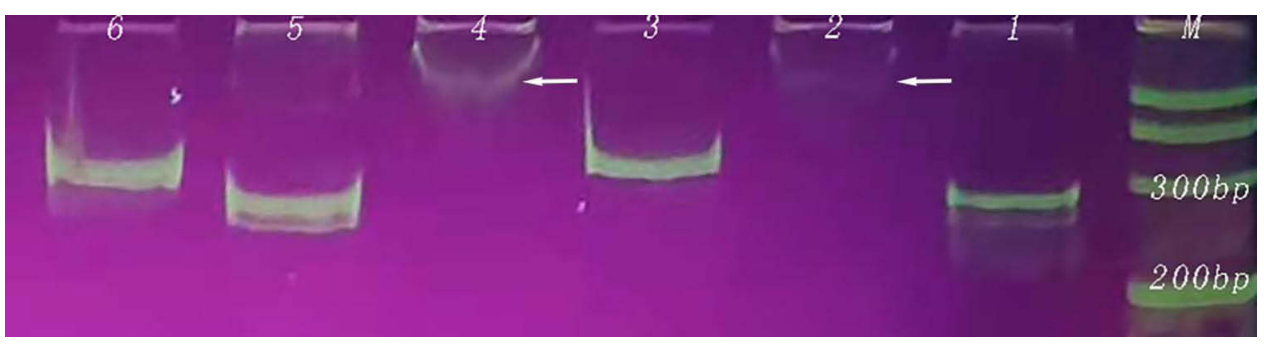

Figure 5 The Rv1453 protein interacts with DNA segments in the qor and Rv1455 promoter regions. 
probe with biotin-labeled on both single strands, and the other two were single-stranded biotin-labeled probes. Bands of DNA probe, migration band resulting from the interaction of DNA probe with protein, and decreased migration band resulting from the addition of unlabeled DNA probe were observable in the positive control group, indicating that the experiment was feasible. The migration of bands due to its binding to Rv1453 protein was observed for both binding sites in the promoter region of the qor gene (Figure 6).

The interaction between Rv1453 protein and Rv1455 double-stranded probe showed that with the increase in protein concentration, the migration band became more and more obvious, while the single-stranded probe decreased. When the unlabeled Rv1455 double stranded probe was added to the reaction solution, the migration bands decreased and the single stranded probe bands disappeared (Figure 7). Due to the decrease in the single-stranded probe, we further investigated whether Rv1453 protein could bind to single stranded probes. It shows that Rv1455 antisense singlestranded probe can also bind to Rv1453 protein, but that the migration band is weak (Figure 8).

Rv1453 protein has the GGDEF_2 and HTH_30 domain and plays a role in transcriptional regulation.
The HTH domain could bind to DNA and be similar to the structure of Bacillus subtilis Pucr transcriptional regulatory protein. The Pucr motif was used to search for binding sites that are located in the promoter region of the $R v 1455$ gene and qor gene, respectively. We found that the DNA segment that can bind to Rv1453 protein in the promoter region of $R v 1455$ gene contains the RNA polymerase binding site within qor gene (Figure 9).

Because the qor gene encodes quinone oxidoreductase, which has the function of reducing free radical damage and oxidative stress, we think that Rv1453 protein could change the level of reactive oxygen species by regulating the transcription of qor gene, thus affecting the susceptibility of the $M$. tuberculosis H37Rv against clofazimine (Figure 10).

\section{Discussion}

The purpose of this study was to investigate the relationship between $R v 1453$ gene and clofazimine resistance in M. tuberculosis. First, the sensitivity of the $R v 1453$ knockout strain, $R v 1453$ complementary strain and $R v 1453$ overexpressed strain to clofazimine were

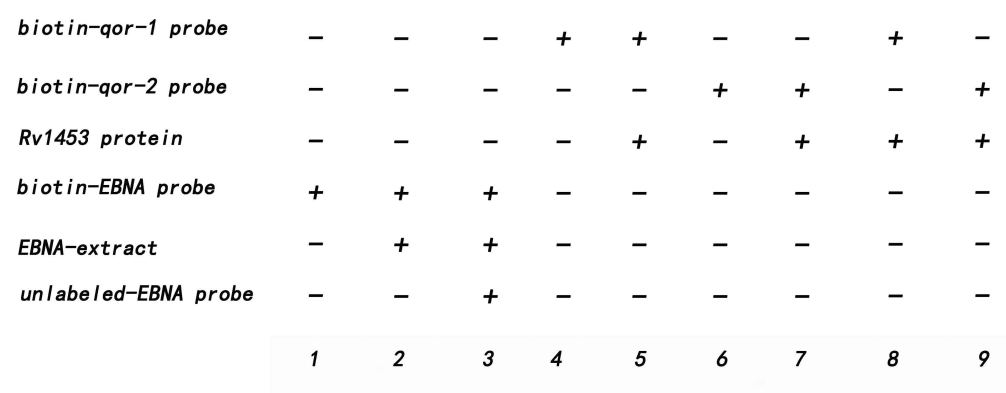

shift band

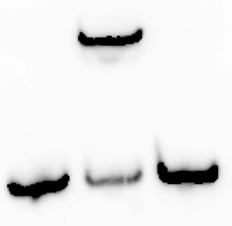

EBNA probe

shift band

free probe

Figure 6 Rvl453 protein interacts with DNA segments from the promoter region of the gor gene. 


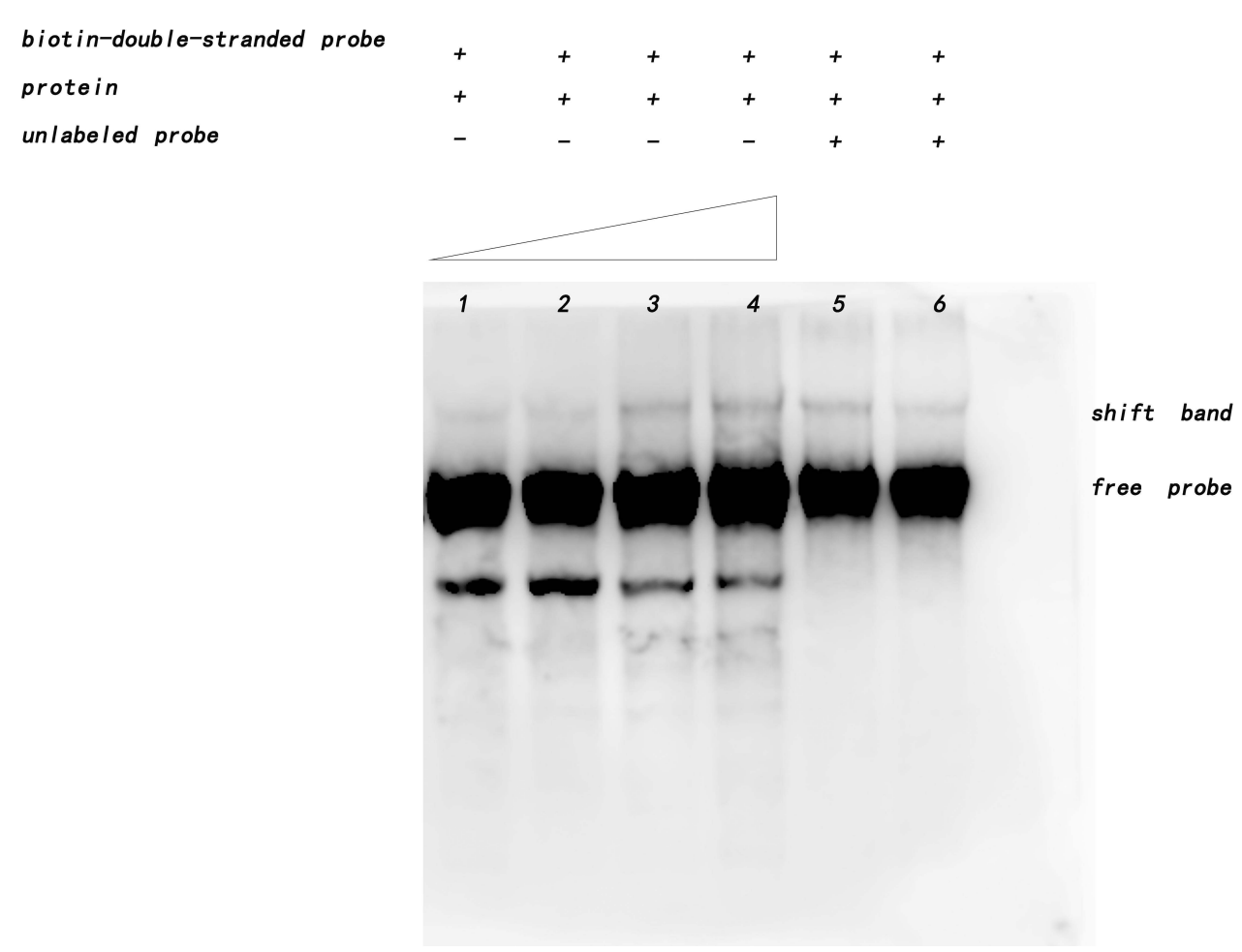

Figure 7 Interaction between Rv1453 protein and Rv/455 double-stranded probe.

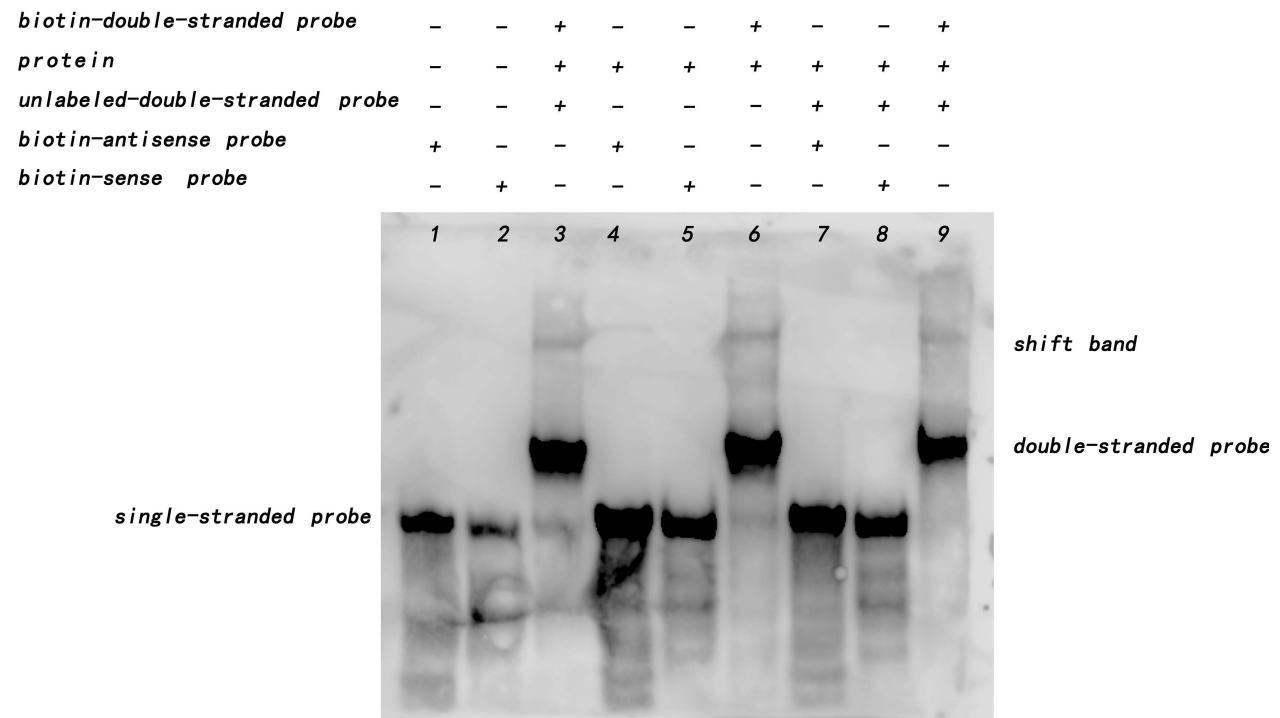

Figure $8 \mathrm{Rvl} 453$ protein interacts with single or double strands of Rv/455.

detected by MABA. It was found that the sensitivity of $R v 1453$ knockout strain to clofazimine was reduced by 4-fold, that of $R v 1453$ overexpressed strain to clofazimine was increased by 4 -fold, and that of Rv1453 complementary strain to clofazimine restored to the same level as M. tuberculosis H37Rv. Therefore, Rv1453 gene is related to the susceptibility of the $M$. tuberculosis to clofazimine. Besides targeted inhibition, antibiotics can also cause cell death by producing reactive oxygen species. ${ }^{17,18}$ The phenazine core is the primary structure of CFZ. Since phenazine molecules are auto oxidizable compounds, they can act as electron acceptors, leading to 
A

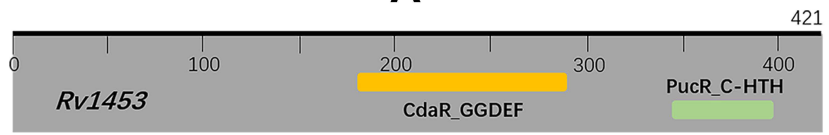

B

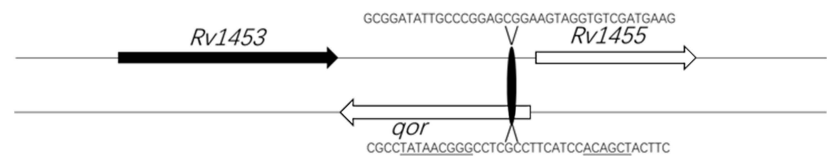

Figure 9 Rv/453 encodes a transcriptional regulatory protein. (A) Functional domain analysis of M. tuberculosis Rvl453 protein. (B) The predicted RNA polymerase and Rvl453 protein binding sites. The predicted RNA polymerase binding sites are underlined. the amount of available ATP decrease. ${ }^{19,20}$ At the same time, intracellular REDOX enables clofazimine to produce reactive oxygen substances. ${ }^{21}$ Therefore, the reduced susceptibility of the $R v 1453$ knockout strain to clofazimine may be due to the decrease in reactive oxygen species. We also found that the MIC of isoniazid and rifampicin could be increased against the $R v 1453$ knockout strain. Isoniazid and rifampicin can significantly increase the formation of hydroxyl radicals. ${ }^{22,23}$ In this study, we found that during the culture of Rv1453 knockout strain, the use of hygromycin could restore MIC to

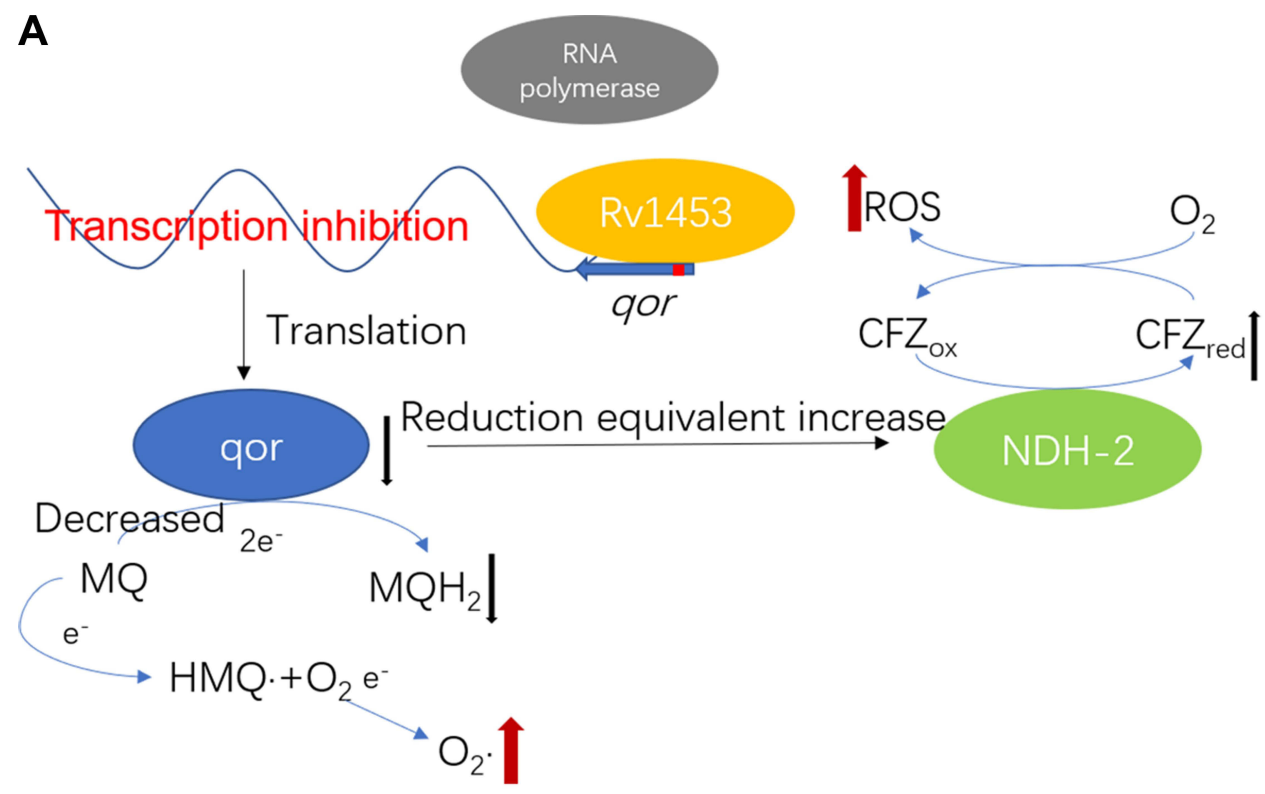

B

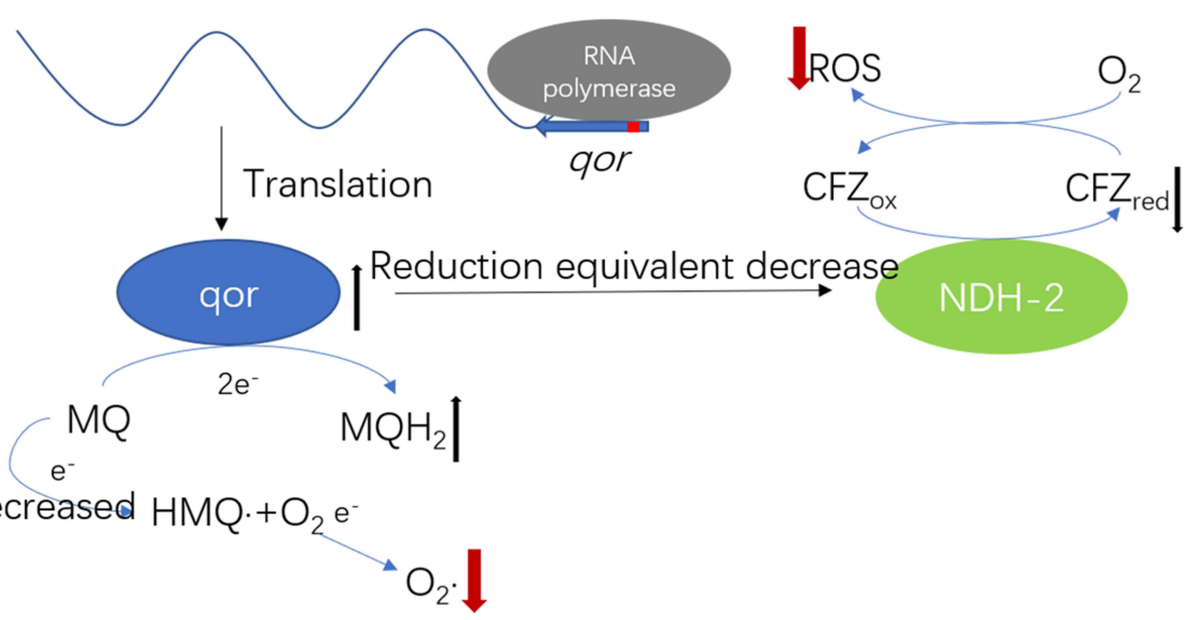

Figure $10 \mathrm{Rv} 1453$ could change the level of reactive oxygen species by regulating the transcription of qor gene, thus affecting the susceptibility of the $M$. tuberculosis $\mathrm{H} 37 \mathrm{Rv}$ against clofazimine. (A) Excessive Rvl453 protein could inhibit the transcription of qor gene and increase the level of reactive oxygen species, which increased the sensitivity of the strain to clofazimine. (B) The deletion of Rvl453 protein increased the transcription of qor gene and decreased the level of reactive oxygen species, which reduced the sensitivity of the strain to clofazimine. 
normal level. The $R v 1453$ gene was replaced with a hygromycin resistance gene in the $R v 1453$ knockout strain, and the hygromycin resistance gene encodes a protein kinase that can phosphorylate hygromycin to make it inactive and thus enable the strain to survive. ${ }^{24}$ However, ATP was consumed in this process. The increase in reactive oxygen species was related to the increased respiration, higher NADH consumption and the lower ATP/ADP ratio. ${ }^{25}$ So, we speculate that the drug resistance may be associated with increased ATP and decreased reactive oxygen species.

Compared with M. tuberculosis H37Rv, Rv1453 overexpressed strain was more sensitive to clofazimine, but had no significant change to isoniazid and rifampicin. This is because CFZ can be reduced by NDH-2 and generate reactive oxygen species in its spontaneous oxidation process. ${ }^{8}$ Reduced quinone reductases lead to increased reduction equivalents, which increase the CFZ's ability to transfer electrons from NDH-2 to oxygen and lead to increased ROS and oxidative stress. ${ }^{26}$

Since $R v 1453$ gene encodes transcriptional regulatory protein, the target genes regulated by $R v 1453$ gene were further analyzed. It was found that the transcription level of qor and $R v 1455$ gene in $R v 1453$ knockout strain and complementary strain were significantly increased, while they were decreased in $R v 1453$ overexpressed strain. This may be due to the fact that the Rv1453 protein expressed by the plasmid has a weak inhibition on the transcription of $R v 1455$ and qor genes, while the knockout of $R v 1453$ had a strong effect on the increase in the transcriptional level of the above two genes. The $R v 1455$ gene encodes a protein of unknown function and the qor gene encodes quinone reductase. Quinone reductase can directly catalyze the two electrons reduction of quinone to hydroquinone, preventing free radical generation during redox by avoiding the one electron reduction of quinone to the semiquinone. ${ }^{14}$ And, it has been reported that lpdA gene, which also encodes quinone reductase, can scavenge reactive oxygen species and reduce the oxidative stress in M. tuberculosis. ${ }^{27,28}$ Inhibition of quinone oxidoreductase activity can increase free radical damage. ${ }^{28}$ In addition, quinone reductase can transfer reducing equivalents to the electron transport chain, which is important for energy production under anaerobic conditions. The hydroquinone reaches the cell membrane to be oxidized to menaquinone, and along with proton translocation across the membrane, establishes an electrochemical proton gradient or proton motive force that drives ATP synthase to produce ATP, favoring $M$. tuberculosis survival. ${ }^{29}$ Since the knockout of $R v 1453$ gene can increase the transcriptional level of qor, it can reduce the susceptibility of the strain to clofazimine by increasing ATP, reducing ROS and oxidative stress.

Although the transcription level of qor gene in the $R v 1453$ complementary strain was significantly increased, its sensitivity to clofazimine was consistent with M. tuberculosis H37Rv. This may be related to the action of clofazimine. As shown in Figure 3, with the increase of clofazimine treatment time, the transcription level of $R v 1453$ in the complementary strain and the overexpressed strain increased significantly. Clofazimine could enhance the transcription level of $R v 1453$. After the addition of clofazimine, the transcriptional levels of qor gene in the Rv1453 complementary strain decreased significantly at $52 \mathrm{H}$. And, the transcriptional levels of qor gene in the Rv1453 overexpressed strain also decreased, although it was not significant. However, we found no decrease in the $R v 1453$ knockout strain. It was considered that the $R v 1453$ knockout strain lacked the transcription regulatory protein encoded by $R v 1453$ gene, which resulted in the uninhibited transcription of $R v 1455$ gene and qor gene.

Nucleic acid-binding proteins have traditionally been divided into two categories: DNA-binding proteins and RNA-binding proteins, but recent studies have found a number of proteins that can bind to both DNA and RNA. Proteins that bind nucleic acids in a sequencespecific manner usually contain one or more well-defined structural motifs (zinc finger, leucine zipper, helix-turnhelix, or helix-loop-helix). ${ }^{30}$ The C-terminal of Rv1453 protein has helix-turn-helix (HTH) domain, which is similar to the structure of Bacillus subtilis Pucr transcriptional regulatory protein, and this domain can bind to target DNA sequence. It has been reported that there is an activation sequence of 5'-WWWCNTTGGTTAA-3' in the upstream of the target gene regulated by PucR, which is called PucR box. So, we predicted the nucleotide sites binding to Rv1453 protein based on the PucR motif and then performed electrophoretic mobility shift assay to study the interaction of Rv1453 protein with the promoter DNA segment of qor gene and Rv1455 gene, respectively. The results showed that Rv1453 protein could bind to the promoter region of $R v 1455$ gene and qor gene. In order to identify the binding site of Rv1453 protein, biotin labelled probe was used for further study. It is found that Rv1453 protein not only binds to Rv1455 double stranded probe but also binds to Rv1455 single stranded probe (antisense strand). Because Rv1455 gene and qor gene are located on 
two different strands with adjacent positions and opposite directions, the DNA sequence of Rv1455 single-strand probe (antisense strand) is consistent with a coding sequence of qor gene, and this sequence contains the predicted RNA polymerase binding site. Therefore, Rv1453 protein binding to this site hinders the binding of RNA polymerase, thus affecting the transcription of qor gene. The levels of ROS and ATP in Rv1453 knockout, complementary and overexpressed strains should be detected in further studies to better elucidate the mechanism of Rv1453 mediated clofazimine resistance in M. tuberculosis.

\section{Conclusion}

As far as we know, we have confirmed that $R v 1453$ gene affected the susceptibility of $M$. tuberculosis to clofazimine for the first time. Further studies showed that the transcriptional regulatory protein encoded by $R v 1453$ gene could bind to the qor gene sequence that contained the RNA polymerase binding site, thereby inhibiting the transcription process. It was preliminarily revealed that the $R v 1453$ knockout strain reduced its susceptibility to clofazimine by increasing the transcription level of qor gene, which provided a new idea for further study on the mechanism of clofazimine resistance.

\section{Acknowledgments}

This work was supported by the National Natural Science Foundation of China (81973367) and Beijing Hospitals Authority Clinical Medicine Development of Special Funding Support (ZYLX202123).

\section{Disclosure}

Miss Yuanyuan Li reports grants from National Natural Science Foundation of China, grants from Beijing Hospitals Authority Clinical Medicine Development of Special Funding Support, during the conduct of the study $\mathrm{Mr}$ Lei Fu reports grants from National Natural Science Foundation of China, grants from Beijing Hospitals Authority Clinical Medicine Development of Special Funding Support, during the conduct of the study. Mrs Weiyan Zhang reports grants from the National Natural Science Foundation of China, grants from Beijing Hospitals Authority Clinical Medicine Development of Special Funding Support, during the conduct of the study. Mrs $\mathrm{Xi}$ Chen reports grants from the National Natural Science Foundation of China, grants from Beijing Hospitals Authority Clinical Medicine
Development of Special Funding Support, during the conduct of the study. Professor Yu Lu reports grants from the National Natural Science Foundation of China, grants from Beijing Hospitals Authority Clinical Medicine Development of Special Funding Support, outside the submitted work. The authors report no conflicts of interest in this work.

\section{References}

1. World Health Organization. Global tuberculosis report 2020. Geneva: World Health Organization; 2020.

2. Tang S, Yao L, Hao X, et al. Clofazimine for the treatment of multidrug-resistant tuberculosis: prospective, multicenter, randomized controlled study in China. Clin Infect Dis. 2015;60(9):1361-1367.

3. Xu HB, Jiang RH, Xiao HP. Clofazimine in the treatment of multidrug-resistant tuberculosis. Clin Microbiol Infect. 2012;18 (11):1104-1110. doi:10.1111/j.1469-0691.2011.03716.x

4. World Health Organization. Global tuberculosis report; 2018.

5. Hartkoorn RC, Uplekar S, Cole ST. Cross-resistance between clofazimine and bedaquiline through upregulation of MmpL5 in Mycobacterium tuberculosis. Antimicrob Agents Chemother. 2014;58(5):2979-2981. doi:10.1128/AAC.00037-14

6. Zhang S, Chen J, Cui P, Shi W, Zhang W, Zhang Y. Identification of novel mutations associated with clofazimine resistance in Mycobacterium tuberculosis. J Antimicrob Chemother. 2015;70 (9):2507-2510. doi:10.1093/jac/dkv150

7. Almeida D, Ioerger T, Tyagi S, et al. Mutations in pepQ confer low-level resistance to bedaquiline and clofazimine in Mycobacterium tuberculosis. Antimicrob Agents Chemother. 2016; 60(8):4590-4599. doi:10.1128/AAC.00753-16

8. Yano T, Kassovska-Bratinova S, Teh JS, et al. Reduction of clofazimine by mycobacterial type $2 \mathrm{NADH}$ : quinone oxidoreductase: a pathway for the generation of bactericidal levels of reactive oxygen species. J Biol Chem. 2011;286(12):10276-10287. doi:10.1074/jbc. M110.200501

9. Lechartier B, Cole ST. Mode of action of clofazimine and combination therapy with benzothiazinones against Mycobacterium tuberculosis. Antimicrob Agents Chemother. 2015;59(8):4457-4463. doi:10.1128/AAC.00395-15

10. Lu P, Heineke MH, Koul A, et al. The cytochrome bd-type quinol oxidase is important for survival of Mycobacterium smegmatis under peroxide and antibiotic-induced stress. Sci Rep. 2015;5:10333. doi:10.1038/srep10333

11. Bolton JL, Trush MA, Penning TM, Dryhurst G, Monks TJ. Role of quinones in toxicology. Chem Res Toxicol. 2000;13(3):135-160. doi:10.1021/tx9902082

12. Oppermann U. Carbonyl reductases: the complex relationships of mammalian carbonyl- and quinone-reducing enzymes and their role in physiology. Annu Rev Pharmacol Toxicol. 2007;47:293-322. doi:10.1146/annurev.pharmtox.47.120505.105316

13. Porté S, Valencia E, Yakovtseva EA, et al. Three-dimensional structure and enzymatic function of proapoptotic human p53-inducible quinone oxidoreductase PIG3. J Biol Chem. 2009;284(25):17 194-17205. doi:10.1074/jbc.M109.001800

14. Siraki AG, Klotz LO, Kehrer JP. Free radicals and reactive oxygen species. Compr Toxicol. 2018;10:262-294.

15. Bardarov S, Bardarov S Jr, Pavelka MS Jr, et al. Specialized transduction: an efficient method for generating marked and unmarked targeted gene disruptions in Mycobacterium tuberculosis, M. bovis BCG and M. smegmatis. Microbiology. 2002;148(Pt 10):3007-3017. doi:10.1099/00221287-148-10-3007 
16. Xu J, Wang B, Hu M, et al. Primary clofazimine and bedaquiline resistance among isolates from patients with multidrug-resistant tuberculosis. Antimicrob Agents Chemother. 2017;61(6). doi:10.11 28/AAC.00239-17.

17. Belenky P, Ye JD, Porter CB, et al. Bactericidal antibiotics induce toxic metabolic perturbations that lead to cellular damage. Cell Rep. 2015;13(5):968-980. doi:10.1016/j.celrep.2015.09.059

18. Dwyer DJ, Belenky PA, Yang JH, et al. Antibiotics induce redox-related physiological alterations as part of their lethality. Proc Natl Acad Sci U S A. 2014;111(20):E2100-E2109. doi:10.1073/ pnas. 1401876111

19. Heikal A, Hards K, Cheung C-Y, et al. Activation of type II NADH dehydrogenase by quinolinequinones mediates antitubercular cell death. $J$ Antimicrob Chemother. 2016;71(10):2840-2847. doi:10.1093/jac/dkw 244

20. Murugesan D, Ray PC, Bayliss T, et al. 2-Mercapto-Quinazolinones as inhibitors of type II NADH dehydrogenase and Mycobacterium tuberculosis: structure-activity relationships, mechanism of action and absorption, distribution, metabolism, and excretion characterization. ACS Infect Dis. 2018;4(6):954-969. doi:10.1021/acsinfecdis. $7 \mathrm{~b} 00275$

21. Cholo MC, Mothiba MT, Fourie B, Anderson R. Mechanisms of action and therapeutic efficacies of the lipophilic antimycobacterial agents clofazimine and bedaquiline. J Antimicrob Chemother. 2017;72(2):338-353. doi:10.1093/jac/dkw426

22. Zeng S, Soetaert K, Ravon F, et al. Isoniazid bactericidal activity involves electron transport chain perturbation. Antimicrob Agents Chemother. 2019;63(3). doi:10.1128/AAC.01841-18.

23. Piccaro G, Pietraforte D, Giannoni F, Mustazzolu A, Fattorini L. Rifampin induces hydroxyl radical formation in Mycobacterium tuberculosis. Antimicrob Agents Chemother. 2014;58(12):7527-75 33. doi:10.1128/AAC.03169-14
24. van den Elzen PJ, Townsend J, Lee KY, Bedbrook JR. A chimaeric hygromycin resistance gene as a selectable marker in plant cells. Plant Mol Biol. 1985;5(5):299-302. doi:10.1007/BF00020627

25. Iqbal IK, Bajeli S, Akela AK, Kumar A. Bioenergetics of mycobacterium: an emerging landscape for drug discovery. Pathogens. 2018;7 (1):24.

26. Lamprecht DA, Finin PM, Rahman MA, et al. Turning the respiratory flexibility of Mycobacterium tuberculosis against itself. Nat Commun. 2016;7:12393. doi:10.1038/ncomms 12393

27. Zheng H, Lu L, Wang B, et al. Genetic basis of virulence attenuation revealed by comparative genomic analysis of Mycobacterium tuberculosis strain H37Ra versus H37Rv. PLoS One. 2008;3(6):e2375. doi:10.1371/journal.pone.0002375

28. Akhtar P, Srivastava S, Srivastava A, Srivastava M, Srivastava BS, Srivastava R. Rv3303c of Mycobacterium tuberculosis protects tubercle bacilli against oxidative stress in vivo and contributes to virulence in mice. Microbes Infect. 2006;8(14-15):2855-2862. doi:10.1016/j. micinf.2006.09.004

29. Sellamuthu S, Singh M, Kumar A, Singh SK. Type-II NADH dehydrogenase (NDH-2): a promising therapeutic target for antitubercular and antibacterial drug discovery. Expert Opin Ther Targets. 2017;21 (6):559-570. doi:10.1080/14728222.2017.1327577

30. Bartas M, Červeň J, Guziurová S, Slychko K, Pečinka P. Amino acid composition in various types of nucleic acid-binding proteins. Int J Mol Sci. 2021;22(2):922. doi:10.3390/ijms22020922
Infection and Drug Resistance

\section{Publish your work in this journal}

Infection and Drug Resistance is an international, peer-reviewed openaccess journal that focuses on the optimal treatment of infection (bacterial, fungal and viral) and the development and institution of preventive strategies to minimize the development and spread of resistance. The journal is specifically concerned with the epidemiology of

\section{Dovepress}

antibiotic resistance and the mechanisms of resistance development and diffusion in both hospitals and the community. The manuscript management system is completely online and includes a very quick and fair peerreview system, which is all easy to use. Visit http://www.dovepress.com/ testimonials.php to read real quotes from published authors. 